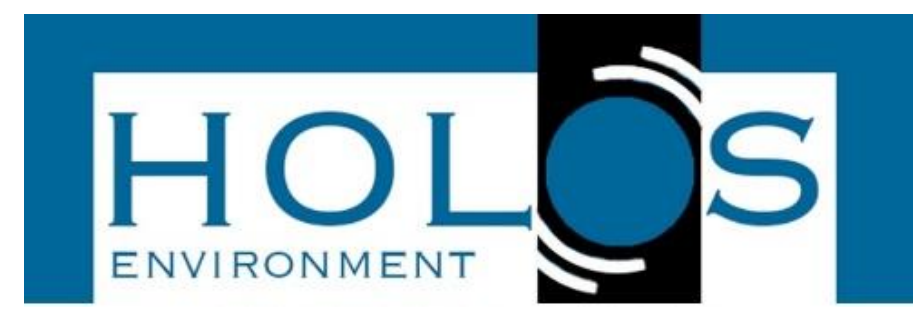

\title{
ROCHA CONTENDO BIOTITA COMO FONTE ALTERNATIVA DE POTÁSSIO PARA FERTILIZANTE APÓS PROCESSAMENTO TÉRMICO COM ADITIVOS
}

\section{ROCK CONTAINING BIOTITE AS AN ALTERNATIVE SOURCE OF POTASSIUM FOR FERTILIZER BY THERMAL PROCESSING WITH ADDITIVES}

\author{
Antônio Clareti Pereira ${ }^{1}$; Marta Rocha dos Santos Gomes²; Sônia Denise Ferreira \\ Rocha $^{1}$
}

Artigo recebido em: 22/11/2018 e aceito para publicação em: 22/11/2019.

DOI: http://dx.doi.org/10.14295/holos.v19i4.12323

Resumo: A biotita é um filossilicato ferromagnesiano que contém potássio. Ela é muito abundante e dispersa em todas as regiões do mundo. Formada secundariamente nos processos metamórficos constitui impureza em muitos processamentos minerais e descartada em bacias de estéreis ou de rejeito. É uma fonte alternativa de potássio para produção de fertilizante, porém, ainda sem exploração comercial em grande escala, para este propósito, por falta de tecnologia que torne o negócio viável economicamente. $\mathrm{O}$ objetivo deste trabalho foi investigar o efeito do processamento térmico, usando aditivos, na solubilização do potássio e impurezas em ácido mineral diluído, de rocha contendo biotita. Foi selecionada uma rocha da província mineral de Carajás-Pará - Brasil, com teor de $9,7 \%$ de $\mathrm{K}_{2} \mathrm{O}$ e $70 \%$ de biotita. O processamento térmico foi executado em duas temperaturas, $800^{\circ} \mathrm{C}$ e $900^{\circ} \mathrm{C}$, durante $6 \mathrm{~h}$, usando dois sistemas de misturas: (biotita+ $+\mathrm{CaCO}_{3}+\mathrm{MgCl}_{2} .6 \mathrm{H}_{2} \mathrm{O}$ ) e (biotita+gesso $+\mathrm{Na}_{2} \mathrm{CO}_{3}$ ). A etapa de lixiviação ácida sulfúrica foi executada em pH mantido entre 2,0 e 3,0 , temperatura de $85^{\circ} \mathrm{C}$, durante $1 \mathrm{~h}$. As extrações, no sistema com cloreto de magnésio $\left(800^{\circ} \mathrm{C}\right)$, chegaram a $63 \%$ do potássio e com solubilização máxima de $5 \%$ de $\mathrm{Fe}$ e Al. As extrações de potássio com gesso ficaram menores, $41 \%$, mostrando tendência de melhor eficiência em temperaturas mais altas do que as escolhidas para este estudo, porém, com licor efluente da lixiviação isento de alumínio e 1,2\% máximo de extração de ferro.

Palavras-chave: Biotita. Processamento térmico. Fertilizante. Potássio.

\begin{abstract}
Biotite is a ferromagnesian phyllosilicate that contains potassium. It is very abundant and scattered all over the world. Usually formed as a secondary product in metamorphic processes, it is considered to be an impurity in many mineral processing. Even though it is an alternative source of potassium, it is still not extracted commercially on a large scale due to the lack of commercial technology that makes the business viable economically. The objective of this work was to investigate the effect of thermal processing, using additives, in the solubilization of potassium and impurities in dilute mineral acid, of rock contains biotite. A rock from the Carajás-Pará-Brazil mineral province, with a content of $9.7 \% \mathrm{~K}_{2} \mathrm{O}$ and $70 \%$ biotite was selected. Thermal processing was performed at two temperatures, $800^{\circ} \mathrm{C}$ and $900^{\circ} \mathrm{C}$, for $6 \mathrm{~h}$ using two systems: (biotite $+\mathrm{CaCO}_{3}+\mathrm{MgCl}_{2} .6 \mathrm{H}_{2} \mathrm{O}$ ) and (biotite + gypsum + $\mathrm{Na}_{2} \mathrm{CO}_{3}$ ). The sulfuric acid leaching step was performed at $\mathrm{pH}$ maintained between 2.0 and 3.0, at 85 ${ }^{\circ} \mathrm{C}$ for $1 \mathrm{~h}$. The extraction in the system with magnesium chloride $\left(800^{\circ} \mathrm{C}\right)$ reached $63 \%$ of the potassium and with solubilization maximum of $5 \%$ of $\mathrm{Fe}$ and Al. The extractions of potassium with gypsum were

\footnotetext{
1 Universidade Federal de Minas Gerais(UFMG) Belo Horizonte, MG. E-mails: claretipereira@gmail.com, sdrocha@demin.ufmg.br) tos@gmail.com)
}

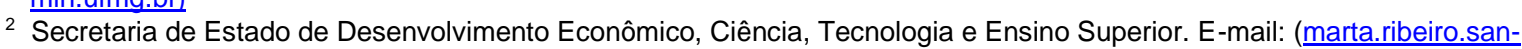


smaller, $41 \%$, showing a trend of better efficiency at higher temperatures than those chosen for this study, however, with effluent liquor from aluminum-free leaching and $1.2 \%$ maximum iron extraction.

Keywords: Biotite. Thermal processing. Fertilizer. Potassium.

\section{INTRODUÇÃO}

Há muitos materiais gerados nos processos de extração mineral que têm agrominerais (corretivos e fertilizantes) e que são descartados como estéreis ou como rejeito do processamento (FRANÇA et al., 2010). Entre eles encontram-se fontes alternativas de potássio como micas e feldspato. A Biotita $\left(\mathrm{K}\left(\mathrm{Mg}, \mathrm{Fe}^{++}\right)_{3} \mathrm{AlSi}_{3} \mathrm{O}_{10}(\mathrm{OH}, \mathrm{F})_{2}\right.$ encontra-se entre estes minerais. Além da disponibilidade em vários rejeitos, há muitos recursos minerais de biotita que podem ser explorados como fonte alternativa de potássio para uso como fertilizante (AGUIAR et al., 2012; OLIVEIRA et al., 2013; CANDIA e GIRARDI, 1979; LOBATO, 2009; OLAREWAJU et al., 2015; TANER et al., 1986; MOORE e CZAMANSKE, 1973; PARAK, 1970; ROSIÈRE \& CHEMALE Jr, 2000).

O potássio é um dos três nutrientes básicos das plantas, conjuntamente com nitrogênio e fósforo. Não existe nenhum substituto para o potássio na agricultura, sendo essencial para manter e expandir a produção de alimentos (MARSCHNER, 1995). Os fertilizantes de liberação lenta do potássio (slow-release fertilizer) usam, principalmente, filossilicatos como matéria-prima provedora do potássio, devido à baixa mobilidade deste potássio em água, ácidos minerais diluídos ou ácidos orgânicos fracos. Para aumentar esta cinética de liberação do potássio, várias pesquisas neste sentido têm sido publicadas (TOKUNAGA, 1991; VALLARELI, 1993; PIZA et al., 2011, ORIOLI Jr e COUTINHO, 2009; MANGRICH et al., 2001). Resultados obtidos nesses estudos indicam que rochas contendo quantidades razoáveis de biotita podem constituir fontes alternativas de potássio para uso agrícola. Estas pesquisas foram enfatizadas entre os anos de 1970 a 1980 (COELHO, 2005). Biotita, leucita, sienito nefelínico, micaxisto, feldspato potássico, cloritaxisto, muscovita e verdete (glauconita) foram alguns dos materiais avaliados (LOPES et al. 1972; FAQUIN, 1982; LEITE, 1985). A prospecção, caracterização geoquímica, definição de métodos de processamento e avaliação agronômica sistemática dessas rochas, resultam na identificação de fontes alternativas eficientes do nutriente, dando origem a um novo grupo de insumos agrícolas (RESENDE et al., 2006). 
Uma maneira de aumentar a disponibilidade do potássio é promover a modificação na estrutura mineral por tratamento térmico (TANNER, 1994). Teixeira et al. (2015) investigaram a liberação de potássio de rochas fonolíticas da região de Poços de Caldas - MG - Brasil por tratamento térmico em temperaturas entre $300^{\circ} \mathrm{C} \mathrm{e}$ $1200^{\circ} \mathrm{C}$. Observaram que, através deste tratamento térmico, foi possível identificar a ocorrência de dois eventos que promovem um aumento da disponibilidade do potássio. Estes eventos estão relacionados com as modificações na composição mineralógica da rocha e, a $1200^{\circ} \mathrm{C}$, com a formação de um material totalmente vítreo. Além disso, observou-se que as mudanças na disponibilidade do potássio são, principalmente, relacionadas às modificações na estrutura do mineral. Mazumder et al. (1993) investigaram a dissolução de potássio em água destilada após tratamento térmico (entre $700^{\circ} \mathrm{C}$ e $850^{\circ} \mathrm{C}$ ) usando uma mistura de arenito contendo glauconita e cloreto de cálcio, variando o tempo de calcinação entre 5 e 180 minutos, granulometria de $300 \mu \mathrm{m}$ a $75 \mu \mathrm{m}$. A lixiviação em água destilada foi executada em temperatura que variou de $40^{\circ} \mathrm{C}$ a $90^{\circ} \mathrm{C}$, conseguindo extrações maiores que $90 \%$ a partir de 10 minutos de lixiviação para calcinações a $850^{\circ} \mathrm{C}$. A relação, para atingir estas extrações, foi de $(1,0: 0,5)$ de arenito e cloreto de cálcio, num tempo de calcinação de 60 minutos.

O objetivo do presente trabalho foi avaliar a solubilização do potássio em solução de diluída de ácido sulfúrico de uma rocha contendo a biotita, após processamento térmico a uma temperatura adequada para liberar este potássio. Mais propriamente avaliar a liberação do potássio da estrutura deste mineral após processamento térmico desta rocha com dois sistemas de mistura distintos: biotita, cloreto de magnésio hexahidratado $\left(\mathrm{MgCl}_{2} .6 \mathrm{H}_{2} \mathrm{O}\right)$ e carbonato de cálcio mineral $\left(\mathrm{CaCO}_{3}\right)$; biotita, gesso químico $\left(\mathrm{CaSO}_{4} .2 \mathrm{H}_{2} \mathrm{O}\right)$ e carbonato de sódio $\left(\mathrm{Na}_{2} \mathrm{CO}_{3}\right)$. Um objetivo secundário é obter, no processo de dissolução em ácido mineral diluído, uma solução carregada com potássio, mas com baixa recuperação de ferro e alumínio.

\section{MATERIAL E MÉTODOS}

\subsection{Caracterização química e mineralógica}

A microscopia eletrônica de varredura (Microscópio Eletrônico de Varredura, marca JEOL, modelo JSM 35C e Espectrômetro de Energia Dispersiva de Raios-X, marca NORAN, modelo Voyager 3050) é aplicada para identificação das fases 
minerais e seus elementos. É utilizado também sistema QEMSCAN 650, equipado com dois espectrômetros EDS (Energy Dispersive X-ray Detector, EDX ou EDS) Bruker SDD (Silicon Drift Detector) 5030, operando a 25kV e 10nA, usando a condição padrão para as medidas, em termos de calibração de sinal de elétrons retro espalhados (BSE - Back-scattered Electron Detector) através de padrões internos de quartzo e cobre metálico.

A quantificação das fases mineralógicas presentes em todas as caracterizações deste trabalho foi através da composição modal. Seguiram as regras de cálculo da Norma CIPW (LOPES, 2012).

Para a análise por ICP/OES a preparação da amostra seguiu os procedimentos abaixo:

$\checkmark$ Fusão por metaborato de lítio: a fusão envolveu a dissolução completa da amostra em fluxo fundido.

$\checkmark$ Espectrometria de Emissão Ótica com Plasma Indutivamente Acoplado Leitura direta - ICP/OES: o equipamento utilizado neste trabalho foi o ICP/OES modelo Perkin Elmer Optima 7300DV.

\subsection{Processamento térmico}

A biotita investigada é proveniente de rocha da região de Carajás-Pará-Brasil. São classificadas como biotitas magmáticas reequilibradas de caráter alcalino e semelhantes às formações em ambientes anorogênicos (CUNHA e DALL'AGNOL, 2012). Para o tratamento térmico foram considerados dois sistemas: $S_{1}=($ rocha + gesso $\left.+\mathrm{Na}_{2} \mathrm{CO}_{3}\right)$ e $\mathrm{S}_{2}=\left(\right.$ rocha $\left.+\mathrm{CaCO}_{3}+\mathrm{MgCl}_{2} \cdot 6 \mathrm{H}_{2} \mathrm{O}\right)$.

$\mathrm{O}$ cloreto de magnésio hexahidratado $\left(\mathrm{MgCl}_{2} .6 \mathrm{H}_{2} \mathrm{O}\right)$ e o gesso $\left(\mathrm{CaSO}_{4} .2 \mathrm{H}_{2} \mathrm{O}\right)$ foram escolhidos por serem subprodutos da indústria de fertilizante, sem aplicação efetiva em longa escala e, portanto, matérias primas com potencial para viabilizar rotas tecnológicas, como estas investigadas, com o propósito de liberar potássio de micas. $\mathrm{O}$ uso do $\mathrm{Na}_{2} \mathrm{CO}_{3}$ está relacionado ao aumento da alcalinidade do sistema no tratamento térmico (MAZUMBER et al., 1993; SANTOS et al., 2015).

As reações no estado sólido são heterogêneas e de cinética lenta, portanto, foi adotado um tempo de residência, para a etapa de processamento térmico de $6 \mathrm{~h}$ (CABRELON et al., 2007), contadas após atingir o valor estabelecido para o ensaio. Considerando a pesquisa de Aitta et al. (1986) foi adotado o tamanho das partículas 
$100 \%<0,15 \mathrm{~mm}$. Este seria o maior valor recomendado para operações de lixiviação em tanque, que é uma possibilidade para a lixiviação ácida. O processamento térmico foi executado em cadinho cerâmico (zirconita) com uma massa inicial de $50 \mathrm{~g}$ de rocha contendo biotita, mantido a $800^{\circ} \mathrm{C}-900^{\circ} \mathrm{C}$, conforme os sistemas da Tabela 1. Foi usado reagente grau analítico (para os aditivos) (Fornecedor Synth).

Tabela 1 - Relação mássica entre os componentes para processamento térmico a $800^{\circ} \mathrm{C}-900^{\circ} \mathrm{C}$

\begin{tabular}{ccc} 
Temperatura $\left({ }^{\circ} \mathbf{C}\right)$ & Sistema & Relação Mássica \\
\hline 800 & rocha $/ \mathrm{CaSO}_{4} / \mathrm{Na}_{2} \mathrm{CO}_{3}$ & $1,0-0,3-0,2$ \\
900 & rocha $/ \mathrm{CaSO}_{4} / \mathrm{Na}_{2} \mathrm{CO}_{3}$ & $1,0-0,3-0,2$ \\
800 & rocha $/ \mathrm{CaCO}_{3} / \mathrm{MgCl}_{2} \cdot 6 \mathrm{H}_{2} \mathrm{O}$ & $1,0-0,2-0,3$ \\
900 & rocha $/ \mathrm{CaCO}_{3} / \mathrm{MgCl}_{2} \cdot 6 \mathrm{H}_{2} \mathrm{O}$ & $1,0-0,2-0,3$ \\
\hline
\end{tabular}

\subsection{Solubilização em ácido mineral}

A extração foi feita em solução ácida sulfúrica em pH mantido entre 2,0-3,0 de tal maneira a evitar a dissolução de $\mathrm{Fe}^{3+}$ (KALINOWSK e SCHWEDA, 1996). Mantida a temperatura em $(85 \pm 5)^{\circ} \mathrm{C}$ por 60 minutos, com agitação e numa relação mássica sólido/líquido inicial de $20 \%$. O cálculo do potássio solúvel foi através da equação:

$\mathrm{K}(\%)=\mathrm{K}_{\mathrm{r}} / \mathrm{K}_{\mathrm{t}} \times 100$, onde $\mathrm{K}_{\mathrm{r}}$ é a massa $\mathrm{K}$ solubilizada e $\mathrm{K}_{\mathrm{t}}$ a massa de potássio da amostra inicial.

\section{RESULTADO E DISCUSSÃO}

\subsection{Caracterização química e mineralógica}

A Tabela 2 apresenta a caracterização química da rocha contendo biotita. Se considerarmos que a magnetita (mineral de ferro) representa somente $2 \%$ da composição mineralógica (Figura 1) pode afirmar que a maior parte do ferro (12,6\% total) está na estrutura da biotita, portanto, mais susceptível à dissolução em meio ácido. $\mathrm{O}$ teor de $\mathrm{K}_{2} \mathrm{O}$ foi de $9,6 \%$. As análises foram por ICP/OES. 
Tabela 2 - Composição química da rocha contendo biotita

\begin{tabular}{cccccccc}
\hline Composto & $\mathrm{Al}_{2} \mathrm{O}_{3}$ & $\mathrm{CaO}$ & $\mathrm{Fe}_{2} \mathrm{O}_{3}$ & $\mathrm{~K}_{2} \mathrm{O}$ & $\mathrm{MgO}$ & $\mathrm{Na}_{2} \mathrm{O}$ & $\mathrm{SiO}_{2}$ \\
\hline$\%$ & 4,3 & 1,0 & 12,6 & 9,6 & 4,3 & 0,3 & 42,2 \\
\hline
\end{tabular}

A mineralogia constituinte da amostra encontra-se no gráfico da Figura 1, com destaque ao alto valor da biotita, $69 \%$.

Figura 1 - Composição mineralógica da rocha conendo biotita

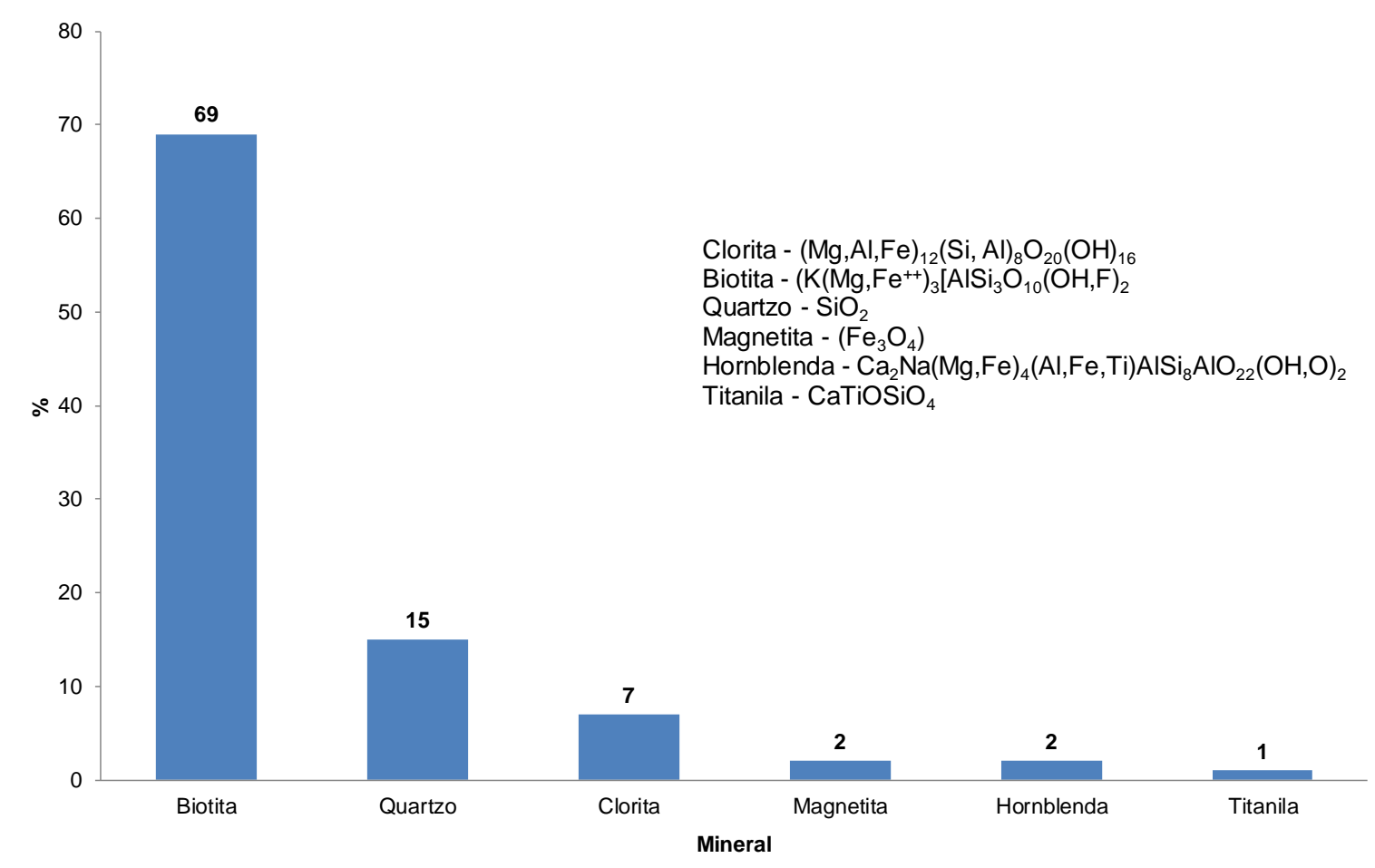

\subsection{Processamento térmico}

Para escolha da temperatura foram feitas simulações de equilíbrio de fases considerando os componentes de entrada. As simulações foram realizadas no aplicativo FactSage módulo Equilib. Para $S_{1}$ (rocha-gesso- $\mathrm{Na}_{2} \mathrm{CO}_{3}$ ), as fases possíveis de se formarem encontram-se na Tabela 3. A escolha de $800^{\circ} \mathrm{C} \mathrm{e} 900^{\circ} \mathrm{C}$ foi em função da formação do composto $\mathrm{K}_{3} \mathrm{Na}\left(\mathrm{SO}_{4}\right)_{2}$ (afitalita), solúvel em água. 
Tabela 3 - Fases presentes para o sistema $\mathrm{S}_{1}$ (rocha; $\mathrm{CaSO}_{4.2} \mathrm{H}_{2} \mathrm{O} ; \mathrm{Na}_{2} \mathrm{CO}_{3}$ )

\begin{tabular}{lccccc} 
Temperatura $\left({ }^{\circ} \mathbf{C}\right)$ & 800 & 850 & 900 & 950 & 1000 \\
\hline $\mathrm{CO}_{2}$ & $\mathrm{G}$ & $\mathrm{G}$ & $\mathrm{G}$ & $\mathrm{G}$ & $\mathrm{G}$ \\
$\mathrm{H}_{2} \mathrm{O}$ & $\mathrm{G}$ & $\mathrm{G}$ & $\mathrm{G}$ & $\mathrm{G}$ & $\mathrm{G}$ \\
$\mathrm{Na}_{2} \mathrm{SO}_{4}$ & $\mathrm{~S}$ & $\mathrm{~S}$ & $\mathrm{~L}$ & $\mathrm{~L}$ & $\mathrm{~L}$ \\
$\mathrm{~K}_{3} \mathrm{Na}\left(\mathrm{SO}_{4}\right)_{2}$ & $\mathrm{~S}$ & $\mathrm{~S}$ & - & - & - \\
$\mathrm{CaSO}_{4}$ & $\mathrm{~S}$ & $\mathrm{~S}$ & $\mathrm{~S}$ & - & - \\
$\mathrm{Mg}_{2} \mathrm{SiO}_{4}$ & $\mathrm{~S}$ & $\mathrm{~S}$ & $\mathrm{~S}$ & $\mathrm{~S}$ & $\mathrm{~S}$ \\
$\mathrm{MgAl}_{2} \mathrm{O}_{4}$ & $\mathrm{~S}$ & $\mathrm{~S}$ & $\mathrm{~S}$ & $\mathrm{~S}$ & $\mathrm{~S}$ \\
$\mathrm{KAISi}_{2} \mathrm{O}_{6}$ & $\mathrm{~S}$ & $\mathrm{~S}$ & $\mathrm{~S}$ & $\mathrm{~S}$ & $\mathrm{~S}$ \\
$\mathrm{CaOMgOSiO}_{2}$ & $\mathrm{~S}$ & $\mathrm{~S}$ & $\mathrm{~S}$ & $\mathrm{~S}$ & $\mathrm{~S}$ \\
$\mathrm{~K}_{2} \mathrm{Ca}_{2}\left(\mathrm{SO}_{4}\right)_{3}$ & - & - & $\mathrm{S}$ & $\mathrm{S}$ & $\mathrm{S}$ \\
$\mathrm{MgOCa}_{2} \mathrm{O}_{2} \mathrm{Si}_{2} \mathrm{O}_{4}$ & - & - & - & $\mathrm{S}$ & $\mathrm{S}$ \\
\hline
\end{tabular}

Para $\mathrm{S}_{2}\left(\right.$ rocha $\left.+\mathrm{CaCO}_{3}+\mathrm{MgCl}_{2} \cdot 6 \mathrm{H}_{2} \mathrm{O}\right)$, as fases possíveis de se formarem encontram-se na Tabela 4, onde G: gás, S: sólido e L: Líquido.

\begin{tabular}{llllll}
\multicolumn{6}{l}{ Tabela 4 - Fases presentes para o sistema $\mathrm{S}_{2}\left(\mathrm{rocha} / \mathrm{CaCO}_{3} / \mathrm{MgCl}_{2} .6 \mathrm{H} 2 \mathrm{O}\right)$} \\
\hline Temperatura $\left({ }^{\circ} \mathrm{C}\right)$ & $\mathbf{8 0 0}$ & $\mathbf{8 5 0}$ & $\mathbf{9 0 0}$ & $\mathbf{9 5 0}$ & $\mathbf{1 0 0 0}$ \\
\hline $\mathrm{CO}_{2}$ & $\mathrm{G}$ & $\mathrm{G}$ & $\mathrm{G}$ & $\mathrm{G}$ & $\mathrm{G}$ \\
$\mathrm{H}_{2} \mathrm{O}$ & $\mathrm{G}$ & $\mathrm{G}$ & $\mathrm{G}$ & $\mathrm{G}$ & $\mathrm{G}$ \\
$\mathrm{HCl}$ & $\mathrm{G}$ & $\mathrm{G}$ & $\mathrm{G}$ & $\mathrm{G}$ & $\mathrm{G}$ \\
$\mathrm{KCl}$ & $\mathrm{L}$ & $\mathrm{L}$ & $\mathrm{L}$ & $\mathrm{G}, \mathrm{L}$ & $\mathrm{G}, \mathrm{L}$ \\
$\mathrm{K}_{2} \mathrm{Cl}_{2}$ & - & - & - & - & $\mathrm{G}$ \\
$\mathrm{Mg}_{2} \mathrm{SiO}_{4}$ & $\mathrm{~S}$ & $\mathrm{~S}$ & $\mathrm{~S}$ & $\mathrm{~S}$ & $\mathrm{~S}$ \\
$\mathrm{KCaCl}_{3}$ & $\mathrm{~S}$ & $\mathrm{~S}$ & $\mathrm{~S}$ & $\mathrm{~S}$ & $\mathrm{~S}$ \\
$\mathrm{CaAl}_{2} \mathrm{Si}_{2} \mathrm{O}_{8}$ & $\mathrm{~S}$ & $\mathrm{~S}$ & $\mathrm{~S}$ & $\mathrm{~S}$ & $\mathrm{~S}$ \\
$\mathrm{MgOCaOSi}_{2} \mathrm{O}_{4}$ & $\mathrm{~S}$ & $\mathrm{~S}$ & $\mathrm{~S}$ & $\mathrm{~S}$ & $\mathrm{~S}$ \\
$\mathrm{MgAl}_{2} \mathrm{O}_{4}$ & $\mathrm{~S}$ & $\mathrm{~S}$ & $\mathrm{~S}$ & $\mathrm{~S}$ & $\mathrm{~S}$ \\
\hline
\end{tabular}

Em função do aparecimento de $\mathrm{KCl}$ nas fases gasosas nas temperaturas acima de $950^{\circ} \mathrm{C}$ e a diminuição da fase sólida $\mathrm{KCaCl}_{3}$ (cloro calcita), solúvel em água, acima de $900^{\circ} \mathrm{C}$, as temperaturas escolhidas também foram $800^{\circ} \mathrm{C}$ e $900^{\circ} \mathrm{C}$.

Para o sistema $\mathrm{S}_{2}$, a escolha do $\mathrm{CaCO}_{3}$ tem a função de diminuir a perda de $\mathrm{HCl}$ devido à decomposição do $\mathrm{MgCl}_{2} .6 \mathrm{H}_{2} \mathrm{O}$ (HUANG et al., 2011). Neste sistema ( $\left.\mathrm{S}_{2}\right)$, 
após o processamento térmico, a $800^{\circ} \mathrm{C}$, as palhetas de biotita apresentaram teores baixos de potássio e teores elevados de cálcio (Figura 2a). Como esses íons ocupam sítios intercamada na estrutura da mica, sugere-se que estão sendo substituídos. Observaram-se também pequenos cristais de $\mathrm{KCl}$ nas bordas das micas (Figura 2b), indicando que o potássio retirado da biotita cristalina está na forma do sal solúvel.

Figura 2 - (a) Palhetas de biotita com teores baixos de K e teores elevados de Ca; (b) Pequenos cristais de $\mathrm{KCl}$ nas bordas das micas

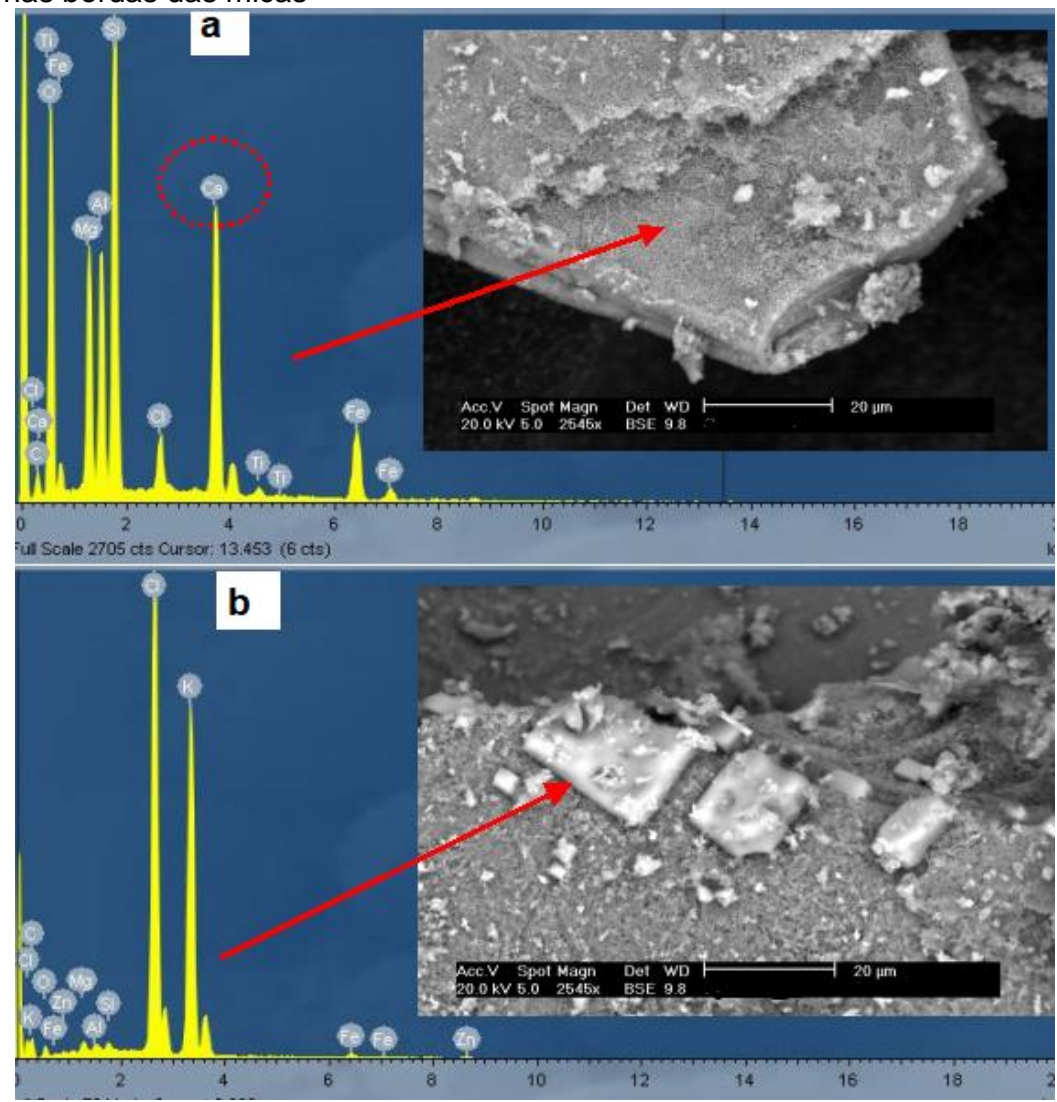

\subsection{Solubilização em ácido mineral}

Após solubilização dos sistemas processados termicamente em ácido sulfúrico (Figura 3) pode se concluir que o ânion cloreto $\left(\mathrm{S}_{2}\right)$ é mais efetivo para liberação do potássio $(63 \%)$ do que o ânion sulfato $\left(S_{1}\right)(41 \%)$ nas temperaturas investigadas $\left(800^{\circ} \mathrm{C}\right.$ e $\left.900^{\circ} \mathrm{C}\right)$. Conclui se também que o íon cloreto começa a perder eficiência acima de $800^{\circ} \mathrm{C}$, inversamente ao sulfato que mostra tendência de aumento na extração de potássio. 
Figura 3 - Solubilização de potássio dos sistemas de misturas $\left(\mathrm{S}_{1}=\right.$ rocha $+\mathrm{CaSO}_{4} .2 \mathrm{H}_{2} \mathrm{O}+\mathrm{Na}_{2} \mathrm{CO}_{3}$ e $\mathrm{S}_{2=} \mathrm{rO}$ cha+ $\mathrm{CaCO}_{3}+\mathrm{MgCl}_{2} 6 \mathrm{H}_{2} \mathrm{O}$ ) processadas termicamente nas temperaturas de $800^{\circ} \mathrm{C}$ e $900^{\circ} \mathrm{C}$. Extração em meio ácido em $\mathrm{pH}$ entre 2,0 e 3,0

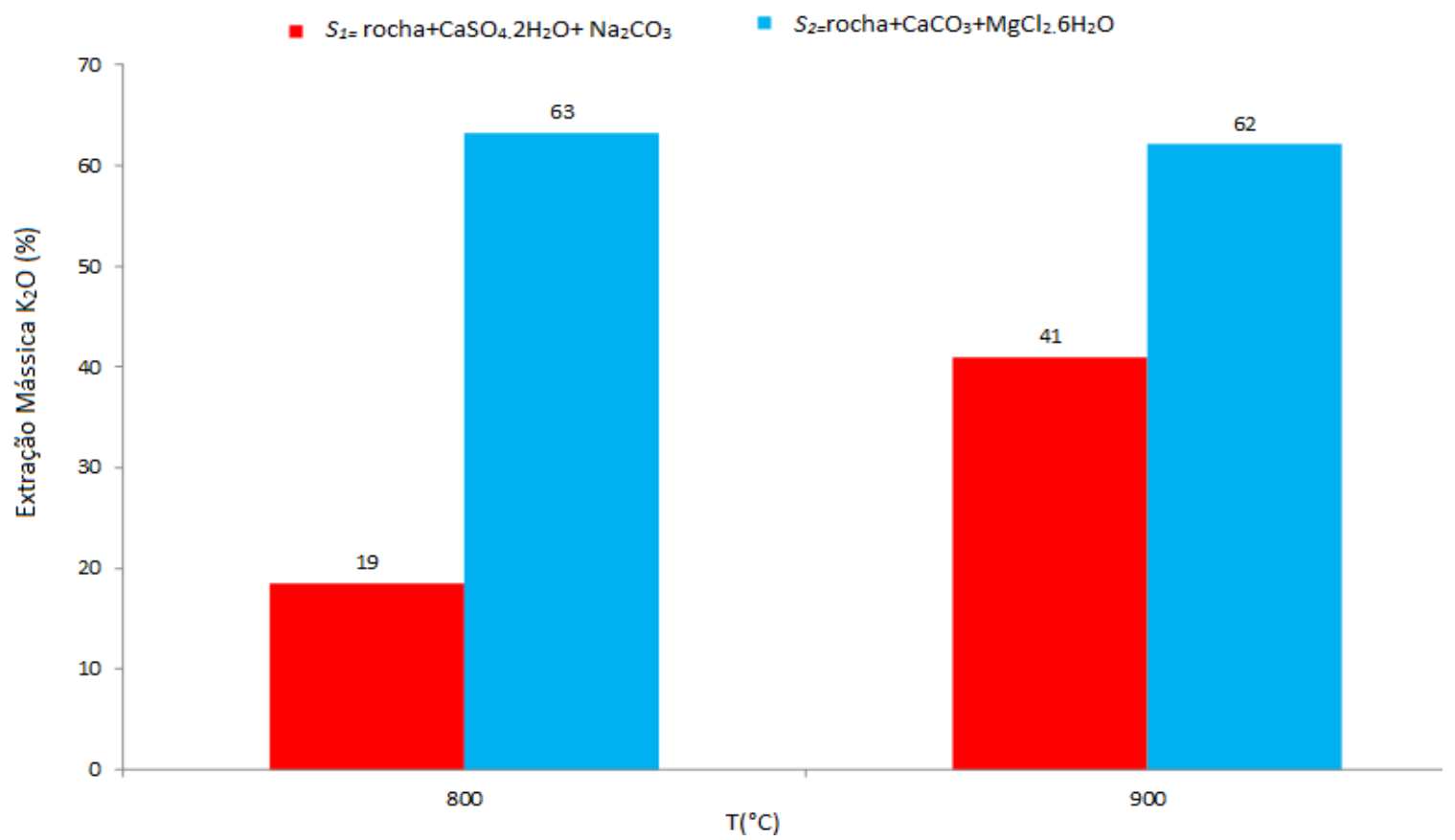

O controle de $\mathrm{pH}$ entre 2,0-3,0 foi efetivo para inibir a dissolução de ferro e alumínio (0\% a 5\% máximo de extração de $\mathrm{Fe}$ e $\mathrm{Al})$, mesmo nas misturas contendo cloreto que formariam compostos solúveis com estes dois elementos (Figura 4).

Figura 4 - Solubilização de $\mathrm{K}$ e Al dos sistemas de misturas $\left(\mathrm{S} 1=\right.$ rocha $+\mathrm{CaSO}_{4} \cdot 2 \mathrm{H}_{2} \mathrm{O}+\mathrm{Na}_{2} \mathrm{CO}_{3}$ e $\mathrm{S}_{2}=\mathrm{rO}_{-}$ cha $+\mathrm{CaCO}_{3}+\mathrm{MgCl}_{2} .6 \mathrm{H}_{2} \mathrm{O}$ ) processadas termicamente nas temperaturas de $800^{\circ} \mathrm{C} \mathrm{e} 900^{\circ} \mathrm{C}$. - Extração em meio ácido em $\mathrm{pH}$ entre 2,0 e 3,0

$=800^{\circ} \mathrm{C}=900^{\circ} \mathrm{C}$

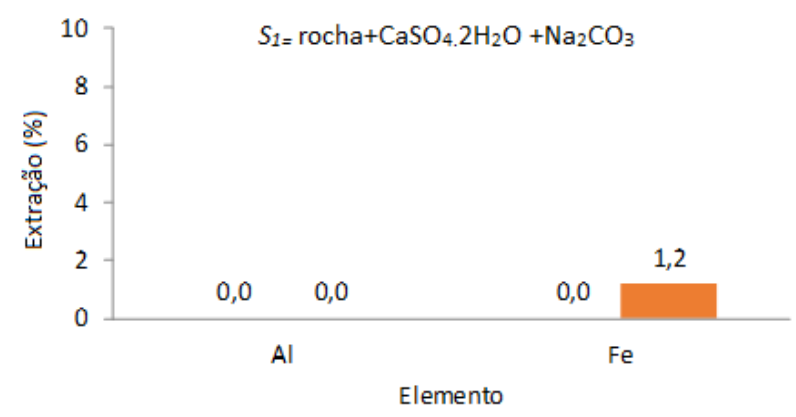

$800^{\circ} \mathrm{C}=900^{\circ} \mathrm{C}$

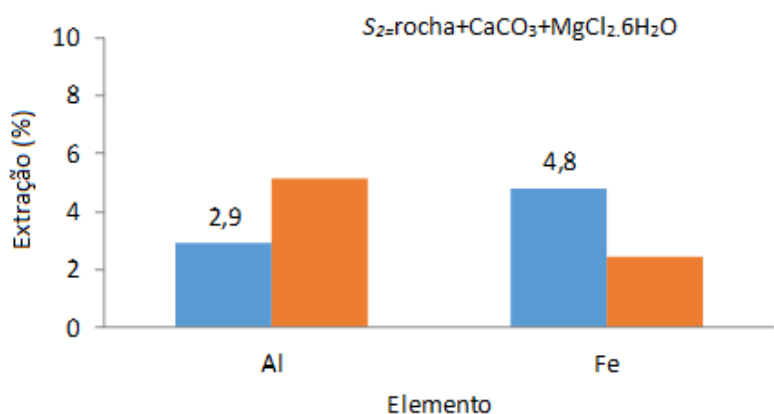

Esta massa calcinada pode ser usada diretamente como um fertilizante de liberação lenta. Os resultados da Figura 4 mostram que há baixa liberação de alumínio, que é um elemento deletério às plantas, e liberações benéficas de potássio (63\%) (NASCIMENTO e LOUREIRO, 2004). A liberação do magnésio foi de $43 \%$ para o $\mathrm{S}_{2}$ a $800^{\circ} \mathrm{C}$. 
A solução resultante do processo de dissolução ficou carregada numa concentração em 2,7g/L de potássio em um estágio de lixiviação. É possível trabalhar com sistemas em contracorrente de tal maneira a aumentar esta concentração.

\section{CONCLUSÃo}

A investigação comprovou que o processamento térmico a $800^{\circ} \mathrm{C}$ foi efetivo para liberação de potássio da estrutura da biotita. O ânion cloreto foi mais efetivo do que o ânion sulfato para a finalidade de liberação do potássio. Há uma tendência de diminuir a extração de potássio para o sistema com cloreto com o aumento da temperatura, no processamento térmico $\left(63 \%\right.$ a $800^{\circ} \mathrm{C}$ e $62 \%$ a $\left.900^{\circ} \mathrm{C}\right)$, enquanto com sulfato tem comportamento oposto $\left(19 \%\right.$ a $800^{\circ} \mathrm{C}$ e $41 \%$ a $\left.900^{\circ} \mathrm{C}\right)$. A extração em ácido mineral diluído, mantendo o pH entre 2,0-3,0, nos sistemas tratados termicamente, deprime, de maneira considerável, a dissolução de ferro e alumínio (0\% a 5\% de extração de $\mathrm{Fe}$ e Al). Com os valores de solubilização do potássio da estrutura da biotita, após processamento térmico, pode se considerar que rocha contendo biotita pode ser aplicada fonte alternativa de potássio para produção de fertilizante.

\section{AGRADECIMENTOS}

Agradecemos o suporte financeiro de Fapemig, CNPq e Capes para este projeto de pesquisa.

\section{REFERÊNCIAS}

AGUIAR. A.P., HORN, A.H, COSTA, A.S.V., LEAL, J.M., ALVES, G.P.P. Estudo da viabilidade em solos agrícolas do uso da biotita-anfibolito/xisto contido nos rejeitos gerados pelos garimpos da província esmeraldífera de Nova Era - MG. Geonomos, v.20, n.1, p.76-80, 2012.

AITTA, E., LESKELA, M., LAJUNEN, L.H.J., JYRKAS, K., SEPPALA, E. Thermal Treatment of Phlogopite and Muscovite with Calcium and Magnesium Compounds. Journal of Chemical Technology and Biotechnology, v.36, p.169-177, 1986.

CABRELON, M. D., ZAUBERAS, R. T., BOSCHI, A. O. Influência da temperatura e do método de mistura na formação do $\mathrm{ZrSiO} 4$ via reação em estado sólido. Cerâmica, v.53, p.8388, 2007. 
CANDIA, M. A. F. \& GIRARDI, V. A. V. Aspectos metamórficos da formação Lafaiete em Morro da Mina, distrito de Lafaiete, MG. Boletim IG, Instituto de Geociências USP, v.10, p.19-30, 1979.

COELHO, A.M. O potássio na cultura do milho. In: Yamada, T. \& Roberts, T.L. (Eds.). Potássio na agricultura brasileira. Piracicaba: Instituto da Potassa \& Fosfato, p.612-658, 2005.

CUNHA, I.R.V. \& Dall'agnol, R. Química mineral em anfibólio e biotita dos granitos da suíte planalto da área de Canaã dos Carajás, província Carajás. In: SEMINÁRIO DE INICIAÇÃO CIENTÍFICA DA UFPA,23., 2012. Livro de resumos [...]. Paraíba, 2012.

FANNING, D.S.; KERAMIDAS, V.Z. \& EL-DESOKY, M.A. Micas. In: DIXON, J.B. \& WEED, S.B., eds. Minerals in soil environments. Madison, Soil Science Society of America, p.551-634, 1989 (SSSA Book Series, 1).

FAQUIN, V. Efeito do tratamento térmico do sienito nefelínico adicionado de calcário dolomítico, na disponibilidade de potássio ao milho (Zea mays L.), em casa de vegetação. Dissertação (Mestrado) - Escola Superior de Agricultura Luiz de Queiroz, Piracicaba. $115 p, 1982$.

FRANÇA, S.C.A., LUZ, A.B., SANTOS, J.S. E BORGES, R.S., Estudo da aplicação de resíduos de vermiculita como fertilizante alternativo de potássio.In: SIMPÓSIO DE MINERAIS INDUSTRIAIS DO NORDESTE, 2., 2010. Anais [...]. p.125-131, Campina Grande-PB, 2010.

HUANG, Q., LU, G., WANG, J. YU, J. Thermal decomposition mechanisms of $\mathrm{MgCl}_{2} \cdot 6 \mathrm{H}_{2} \mathrm{O}$ and $\mathrm{MgCl}_{2} \cdot \mathrm{H}_{2} \mathrm{O}$. Journal of Analytical and Applied Pyrolysis, v.91, p.159-164, 2011.

KALINOWSKI, B.E. \& SCHWEDA, P. Kinetics of muscovite, phlogopite, and biotite dissolution and alteration at $\mathrm{pH} 1-4$, room temperature. Geochimica et Cosmochimica Acta, v.60, n.3, p.367-385, 1996.

LEITE, P.C. Efeitos de tratamentos térmicos em misturas de verdete de Abaeté, fosfato de Araxá e calcário magnesiano, na disponibilidade de potássio e fósforo. Dissertação (Mestrado) - Escola Superior de Agricultura de Lavras, Lavras, 1985. 146 p.

LOBATO, E. A. Mineração Brasileira: perfil da Mica. Ministério de Minas e Energia-MME, Secretaria de Geologia, Mineração e Transformação Mineral-SGM. Relatório Técnico 51, 2009.

LOPES, A.S.; FREIRE, J.C.; AQUINO, L.H., FELIPE, M.P. Contribuição ao estudo da rocha potássica - Verdete de Abaeté (Glauconita) para fins agrícolas. Agros, v.2, p.32-42, 1972.

LOPES, C.L. Composições mineralógicas virtuais em rochas ígneas. Universidade de Évora, 2012. 30p.

MANGRICH, A., TESSARO, L., DOS ANJOS, A., WYPYCH, F., SOARES, J. A slow-release $\mathrm{K}+$ fertilizer from residues of the Brazilian oil-shale industry: synthesis of kalsilite-type structures. Environmental geology, v.40, n.8, p.1030-1036, 2001.

MARSCHNER, H. Mineral nutrition of higher plants. London, Academic Press, 1995. 889 p. 
MAZUMDER, A.K., SHARMA, T., RAO, T.C. Extraction of potassium from glauconitic sandstone by the roast-leach method. International Journal of Mineral Processing, v.38, p.111-123, 1993.

MOORE, W.J \& CZAMANSKE, G.K. Compositions of biotites from unaltered and altered monzonitic rocks in the Bingham Mining District, Utah. Economic Geology, v.68, p.269-274, 1973.

NASCIMENTO, M. \& LOUREIRO, F.E.L.. Fertilizantes e sustentabilidade: o potássio na agricultura brasileira, fontes e rotas alternativas. Rio de Janeiro: CETEM/MCT, 66 p. (Série Estudos e Documentos, 61), 2004.

OLAREWAJU, A.O, AYODELE, B.E, GBENGA, B.S. Geometallurgical Evaluation of Itapa Ekiti Feldspathic-Biotite Ore Deposit for Effective Processing and Extraction. International Journal of Mining Engineering and Mineral Processing, v.4, n.1, p.1-7, 2015.

OLIVEIRA, S.F. CUNHA, A.L.C, MENDES V.A. Contexto geológico e estrutural da formação Seridó, nas pedreiras do "preto matriz", município de Currais Novos/RN- província Borborema. Revista Brasileira de Mineração e Meio Ambiente, v.3, n.1, p.32-40, 2013.

ORIOLI Jr \& V., COUTINHO, E.L.M. "Effectiviness of Fused Magnesium Potassium Phosphate for Marandu Grass", Revista Brasileira de Ciência do Solo, v. 33, p.1855-1862, 2009.

PARAK, T. Kiruna iron ores are not "intrusive-magmatic ores of the Kiruna type". Economic Geology, v.70, p.1242-1258, 1975.

RESENDE, A.V., MARTINS, E.S., OLIVEIRA, C.G., SENA, M.C., MACHADO, C.T.T., KINPARA, D.I., OLIVEIRA Filho, E.C. Suprimento de potássio e pesquisa de uso de rochas "in natura" na agricultura brasileira. Espaço \& Geografia, v.9, n.1, 2006.

ROSIÈRE, C.A. \& CHEMALE Jr, F., Itabiritos e minérios de ferro de alto teor do quadrilátero ferrífero - uma visão geral e discussão. Geonomos, v.8, n.2, p-27-43, 2000.

SANTOS, W.O.; MATTIELLO, E.M.; COSTA, L.M.; ABRAHÃO, W.A.P.; NOVAIS, R.F.; CANTARUTTI, R.B. Thermal and chemical solubilization of Verdete for use as potassium fertilizer. International Journal of Mineral Processing, v.140, p.72-78, 2015.

TANNER Jr., J. T., "Mica", Industrial Minerals and Rocks, 6. ed. Ed. Donald D. Carr, 1994.

TANER, M.F., TRUDEL, P., PERRAULT, G. Geoghimie de la biotite associée à certains gisements d'or de Val d'or, Malartic et Chibougamau, Québec. Canadian Mineralogist, v.24, p.761-774, 1986.

TEIXEIRA, A.M.S.; GARRIDO, F.M.S.; MEDEIROS, M.E.; SAMPAIO, J.A. Estudo do comportamento térmico da rocha fonolito com fins à produção de fertilizantes. Holos, v.5, 2015.

TOKUNAGA, Y. Potassium silicate: a slow-release potassium fertilizer. Fertilizer Research, v.30, p.55-59, 1991.

PIZA, P.A.T.; Bertolino, L.C., Silva, A.A.S., Sampaio, J.A., LUZ, A.B. Verdete da região de Cedro do Abaeté (MG) como fonte alternativa para potássio. Geociências, v.30, p.345-356, 2011. 
VALLARELI. J.V. Ardósias verdete de cedro do abaeté na produção de termofosfato potássico fundido e sua eficiência agronômica. Anais da Academia Brasileira de Ciências, v. 31, p. 363-375, 1993. 